\title{
EXCITATION ENERGY TRANSPORT BETWEEN THE IONIC FORMS OF RHODAMINE B IN VISCOUS SOLUTIONS*
}

\author{
P. BoJARSKI ${ }^{a}$ AND L. KUlAK ${ }^{b}$ \\ ${ }^{a}$ University of Gdańsk, Institute of Experimental Physics \\ Wita Stwosza 57, 80-952 Gdańsk, Poland \\ ${ }^{b}$ Technical University of Gdańsk \\ Department of Technical Physics and Applied Mathematics \\ Narutowicza 11/12, 80-952 Gdańsk, Poland
}

(Received July 23, 1998)

Dedicated to Prof. Dr. C. Bojarski on the occasion of his 75th birthday

\begin{abstract}
Excitation energy transport between zwitterion and cationic form of rhodamine B in glycerol-ethanol mixture is studied. Concentration course of the quantum yield exhibits a rapid drop at intermediate concentrations, where no dimers are formed contrary to the results of measurements carried out for solutions with a trace amount of acid or base in which single ionic forms are adopted. To describe properly such concentration behavior of the quantum yield forward and reverse, nonradiative excitation energy transport between the forms is considered. This task is performed by comparing experimental data with Monte-Carlo simulations.
\end{abstract}

PACS numbers: 31.15.-p, 33.50.-j

\section{Introduction}

Over past several years rapid progress in the theoretical description of excitation energy transport in two-component systems has been made by taking into account not only forward, but also reverse energy transfer [1-9]. This effect has been confirmed experimentally in model donor-acceptor systems [9-13] as well as in monomer-fluorescent dimer systems [14-16]. In those systems it modifies significantly the concentration courses of luminescent observables, but it is relatively weak as compared to the forward transfer and energy migration. However, in systems adopting different ionic forms, it seems to be of a much more comparable strength, as all the absorption and fluorescence spectra overlap strongly

*The results of this paper were initially presented at The Jabtonski Centennial Conference on Luminescence and Photophysics, July 23-27, 1998, Torun', Poland. 
and different ionic forms have comparable quantum yields. As such systems play sometimes an important role in nature, it seems necessary to study the mechanism of excitation energy transport in them.

The task of this paper is to analyze the effect of energy transport on the concentration courses of the quantum yield in glycerol-ethanol solutions of rhodamine $\mathrm{B}$ (RB), which adopts normally two ionic forms: a zwitterion and a cationic one. Previous studies of photophysical properties of $\mathrm{RB}$ solutions revealed the fact that the base-acid equilibrium shifts from the zwitterion to the cationic form with the increase in concentration and that at very high concentrations the changes in RB characteristics result from dimerization [17-26]. Since all the absorption and fluorescence spectra of both molecular forms overlap strongly, it is necessary however to consider excitation energy transport within and between the sets of both species terminated by energy trapping at highest concentrations by dimers. These transport phenomena exhibit generally a strong influence on luminescence characteristics but until now the quantitative explanation of concentration anomalies observed in such systems $[19,22]$ has not been undertaken. The task of our paper is achieved by comparing between the concentration courses of the experimental quantum yield and those obtained by means of the Monte-Carlo simulation. Since the fluorescence is emitted by both forms in a similar spectral region a comparison to recently developed self-consistent diagrammatic theory (SCDM), which assumes that only donors of energy are excited and only light from their fluorescence characteristics is recorded [6-9], would be complicated. Suitable adaptations to this theory are in progress and an appropriate study will be reported separately.

\section{Monte-Carlo simulation}

In a simulation, $N$ donors of concentration $C_{\mathrm{D}}$ and $M$ acceptors of concentration $C_{\mathrm{A}}$, are randomly distributed in a three-dimensional cube. The effect of the finite size of the system is reduced by introducing periodic boundary conditions (the cube is surrounded by replicas of itself) with minimum image convention (the molecule interacts with another molecule or with its periodic image). The concentration course of the quantities of interest is obtained by rescaling critical radii for energy transfer and keeping the length of the cube edge equal to 1. The pseudo-random number generator (mixed congruential generator with the period of $2^{32}$ ), which passed several statistical tests was also verified by checking the simulated statistical clusters concentration against the analytically expected value. The simulated configurations were sampled until the relative variance of the luminescent observables attained less than $0.1 \%$.

\subsection{Step by step Monte-Carlo method}

The "step by step" Monte-Carlo simulation method consists in uses of the random number generator for the cyclic formulation of answers to two questions: when any of the assumed luminescent processes will take place in the simulated system, and what kind of process it will be. The simulation algorithm includes the following steps: 
$1^{\circ}$ The coordinates of a primarily excited molecule are determined (using the random number generator). This molecule can be deactivated through the following processes:

$\left(\mathrm{P}_{1}\right)$ process 1: $D^{*} \rightarrow D$, photon emission or nonradiative energy transition, with the rate $1 / \tau_{O D}$;

$\left(\mathrm{P}_{2}\right)$ process $2: D^{*}+D \rightarrow D+D^{*}$, energy migration (energy transfer to the molecules of the same kind), with the transfer rate $w_{j i}^{D D}$;

$\left(\mathrm{P}_{3}\right)$ process $3: D^{*}+A \rightarrow D+A^{*}$, nonradiative energy transfer from the excited donor to an acceptor, with the transfer rate $w_{j i}^{D A}$;

$2^{\circ}$ If $i$-th donor molecule is excited, the values of the following total transfer rates

$$
c_{1 i}=\frac{1}{\tau_{0 M}}, \quad c_{2 i}=\sum_{j=1, i \neq j}^{N} w_{i j}^{M M}, \quad c_{3 i}=\sum_{j=N+1}^{N+M} w_{i j}^{M D}
$$

have to be calculated. Otherwise, when $i$-th acceptor is excited, the values of

$$
c_{1 i}=\frac{1}{\tau_{0 D}}, \quad c_{2 i}=\sum_{j=1, i \neq j}^{N} w_{j i}^{D D}, \quad c_{3 i}=\sum_{j=N+1}^{N+M} w_{j i}^{D M}
$$

are calculated.

$3^{\circ}$ The time, at which any of the investigated processes occur (cp. step $1^{\circ}$ ), is calculated by inverting the distribution function of the probability $p_{i}\left(t, P_{k}\right) \mathrm{d} t$ that if at time $t$ the $i$-th molecule is excited, then the process $P_{k}$ appears in the time interval $(t, t+\mathrm{d} t)$

$$
p_{i}(t)=\sum_{k=1}^{3} p\left(t, P_{k}\right)=c_{i} \exp \left(-c_{i} t\right),
$$

where

$$
c_{i}=c_{1 i}+c_{2 i}+c_{3 i} .
$$

For this purpose the random number $r_{1 i}$ is generated and the time at which any process takes place is obtained by inverting the distribution function of probability $p_{i}\left(t, P_{k}\right)$,

$$
\int_{0}^{t_{i}} p_{i}(t) \mathrm{d} t=r_{1 i}, \quad \text { i.e. } \quad t_{i}=-\left(\frac{1}{a_{i}}\right) \ln r_{1 i} .
$$

The same procedure can be applied to the excited acceptor. In this case all constants $c_{j i}$ should be replaced by $c_{j i}^{\prime}$.

$4^{\circ}$ In this step it is determined which process took place at the time $t_{i}$. By generating the next random number, $r_{2 i}$, such a value of index $k$ can be found for which the following inequality is satisfied:

$$
\sum_{j=1}^{k-1} c_{i j}<r_{2 i} c_{i} \leq \sum_{j=1}^{k} c_{j i}, \quad k=1,2,3 .
$$

If $k=1$, then the activated molecule is deactivated by a photon emission or nonradiative transition and it means that this pass of simulation is finished. If $k=2$ or $k=3$, the energy migration or transfer process takes. place, and it is 
necessary to determine currently activated molecule. For this reason, the third random number, $r_{3 i}$, is generated and the value of index $n$ is found which fulfills one of the following inequalities:

$$
\sum_{j=1}^{n-1} w_{j i}^{M M}<r_{3 i}\left(c_{1 i}+c_{3 i}\right) \leq \sum_{j=1}^{n} w_{j i}^{M M}, \quad n \leq N
$$

or

$$
\sum_{j=1}^{N} w_{j i}^{M M}+\sum_{j=1}^{n-1} w_{j i}^{M D}<r_{3 i}\left(c_{1 i}+c_{3 i}\right) \leq \sum_{j=1}^{N} w_{j i}^{M M}+\sum_{j=N+1}^{n} w_{j i}^{M D}, \quad n \geq N,
$$

where $n$ is the number of next activated donor or acceptor molecule. Then, after inserting the value of $n$ for the index $i$, the simulation goes to step $2^{\circ}$. The simulation run is finished when after several migration or transfer energy acts the process with $k=1$ occurs in step $4^{\circ}$ (photon emission or nonradiative energy transition). After that a new simulation runs (i.e., for a new donor and acceptor spatial configuration). In the case when an acceptor is excited, the number of subsequently excited molecule, $k$, is determined from the following relations:

$$
\sum_{j=N+1}^{n-1} w_{j i}^{D D}<r_{3 i}\left(c_{1 i}^{\prime}+c_{3 i}^{\prime}\right) \leq \sum_{j=N+1}^{n} w_{j i}^{D D}, \quad n \leq N+M
$$

and

$$
\sum_{j=N+1}^{N+M} w_{j i}^{D D}+\sum_{j=1}^{n-1} w_{j i}^{D M}<r_{3 i}\left(c_{1 i}^{\prime}+c_{3 i}^{\prime}\right) \leq \sum_{j=N+1}^{N+M} w_{j i}^{D D}+\sum_{j=1}^{n} w_{j i}^{D M}, \quad n \leq N .
$$

The relative donor (acceptor) quantum yield is calculated by dividing the number of simulation runs finished with the donor (acceptor) emission by the number of all runs. At the highest concentration in the system studied dimers appear. They play a role of perfect traps for excitation energy and their presence is taken into account in the simulation in a similar manner as described above with the difference that they cannot transfer excitation energy back to any monomer. Therefore, if the excitation was trapped by a dimer, the specific simulation run was finished.

\section{Experimental procedures}

Analytically pure rhodamine B (Aldrich) was additionally purified by multiple crystallization. Glycerol and ethanol used were analytically pure and water free. To minimize the influence of secondary effects on the fluorescent observables the optical density, $D$, of each sample was kept lower than 0.1 [27] and at concentrations $C>0.04 \mathrm{M}$ it did not exceed 0.2 . Three series of systems differing in $\mathrm{pH}$ were prepared (sixteen samples of different dye concentrations in each system). Different $\mathrm{pH}$ was produced by adding a trace of $\mathrm{NaOH}$ to system I and $\mathrm{HCl}$ to system II. These two systems play a role of reference in the experiment of interest. System III, in which pH changes with concentration was prepared without any additives.

In particular, the dimerization constants used in the analysis of system III are calculated based on the measurements performed for systems I and II. Table I lists 
TABLE I

Basic physical properties of the systems studied $\left(n-\right.$ refractive index, $\eta_{0}-$ fluorescence quantum yield, $K_{d}$ - equilibrium constant for dimerization, $K_{b a}-$ base-acid equilibrium constant).

\begin{tabular}{|c|c|c|c|c|c|c|c|}
\hline System & Solvent & $\begin{array}{c}\text { Addi- } \\
\text { tives }\end{array}$ & $\mathrm{pH}$ & $n$ & $\eta_{0}$ & $\begin{array}{c}K_{d} \\
{[1 / \mathrm{M}]} \\
\end{array}$ & $\begin{array}{c}K_{b a} \\
{[1 / \mathrm{M}]}\end{array}$ \\
\hline I & \multirow{3}{*}{$\begin{array}{c}90 \% \\
\text { glycerol } \\
10 \% \\
\text { ethanol }\end{array}$} & $\mathrm{NaOH}$ & $7.5 \pm 0.1$ & \multirow[t]{3}{*}{1.46} & $0.75 \pm 0.05$ & 2.7 & - \\
\hline II & & $\mathrm{HCl}$ & $4.0 \pm 0.1$ & & $0.5 \pm 0.04$ & 1 & - \\
\hline III & & - & $\begin{array}{c}\text { decreasing } \\
\text { with } C \\
(7.5 \rightarrow 4.0) \\
\pm 0.1\end{array}$ & & $0.75 \pm 0.05$ & - & 1350 \\
\hline
\end{tabular}

basic parameters of the systems studied. To calculate the dimerization constants of both forms we have written software based on a modified Förster-Levshin method, which exploits the concentration changes in absorption spectra and a law of mass action [28-30]. Similar values as specified in Table I were also obtained from concentration quenching experiment performed for systems I and II. The base-acid equilibrium constant was calculated in a similar manner by analyzing concentration changes in absorption spectra of system III, after some minor changes in the program. In the latter case we used a limited concentration range, to avoid the distortion by dimers $(C<0.01 \mathrm{M})$.

Absorption spectra were measured using a computerized Carl-Zeiss Specord M-40 spectrophotometer. Fluorescence spectra and quantum yields were measured upon frontal excitation and observation using the apparatus described separately [31] and the results were corrected for the spectral sensitivity of the equipment. The observables were also corrected for the spatial anisotropy distribution of the polarized fluorescence $[32,33]$. Emission anisotropy was measured using a single-photon-counting apparatus described separately with an accuracy of 0.003 $[34,11]$. Measurements were carried out at room temperature.

\section{Results and discussion}

Figure 1 shows the absorption and fluorescence spectra of both forms for low concentrations and at room temperature. Strong overlap can be seen between all pairs of absorption and fluorescence spectra, which means that the energy transport can take place as a multistep process within and between both forms in forward (base to acid) and reverse (acid to base) directions. Table II gives the values of energy transport parameters for the system investigated.

Figure 2 presents the results of a concentration course of the quantum yield for system III (no additives). It is seen that the quantum yield course exhibits a significant anomaly at intermediate concentrations, where virtually no dimers are formed (cp. Table III) and for $C \geq 0.01 \mathrm{M}$ a rapid drop in its values due to the concentration quenching by dimers is seen. The relative quantum yield drops in 


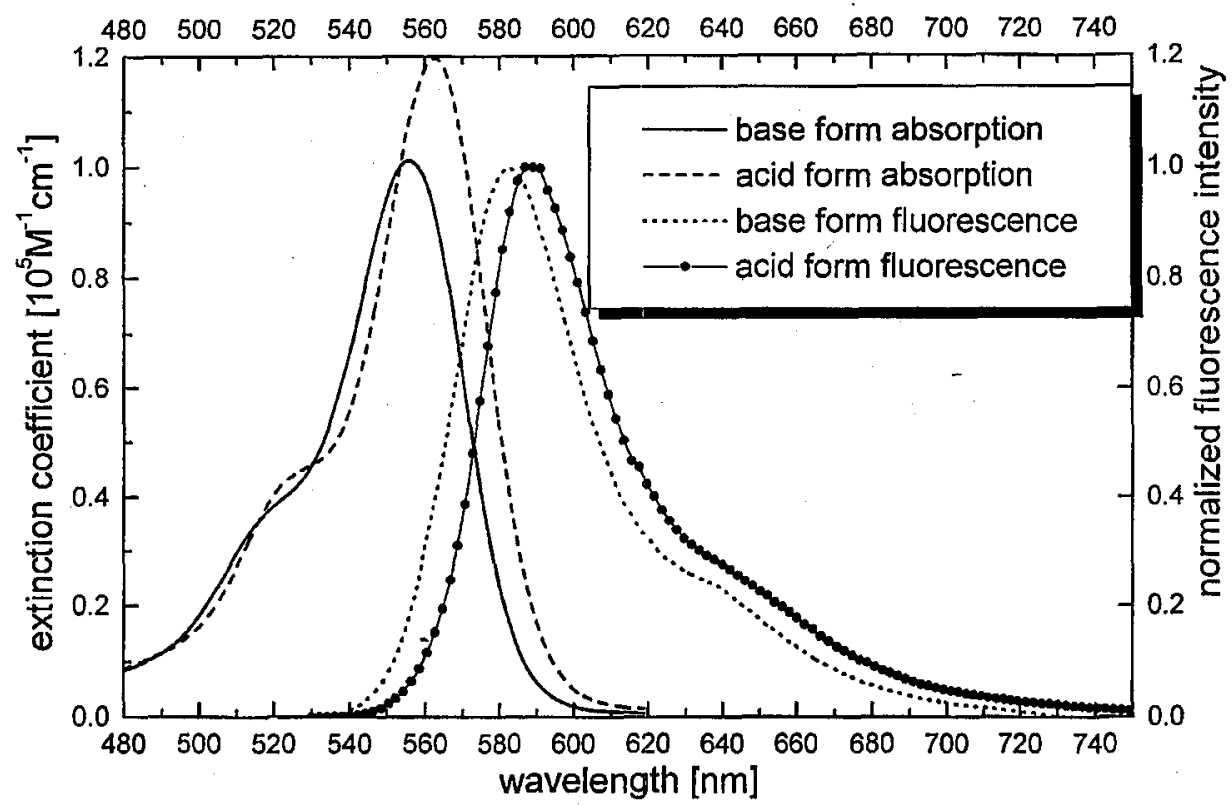

Fig. 1. Absorption and fluorescence spectra of RB ionic forms measured for low concentrations of the dye at room temperature. The spectra of zwitterion and cationic forms were obtained by adding trace amounts of $\mathrm{NaOH}$ and $\mathrm{HCl}$, respectively.

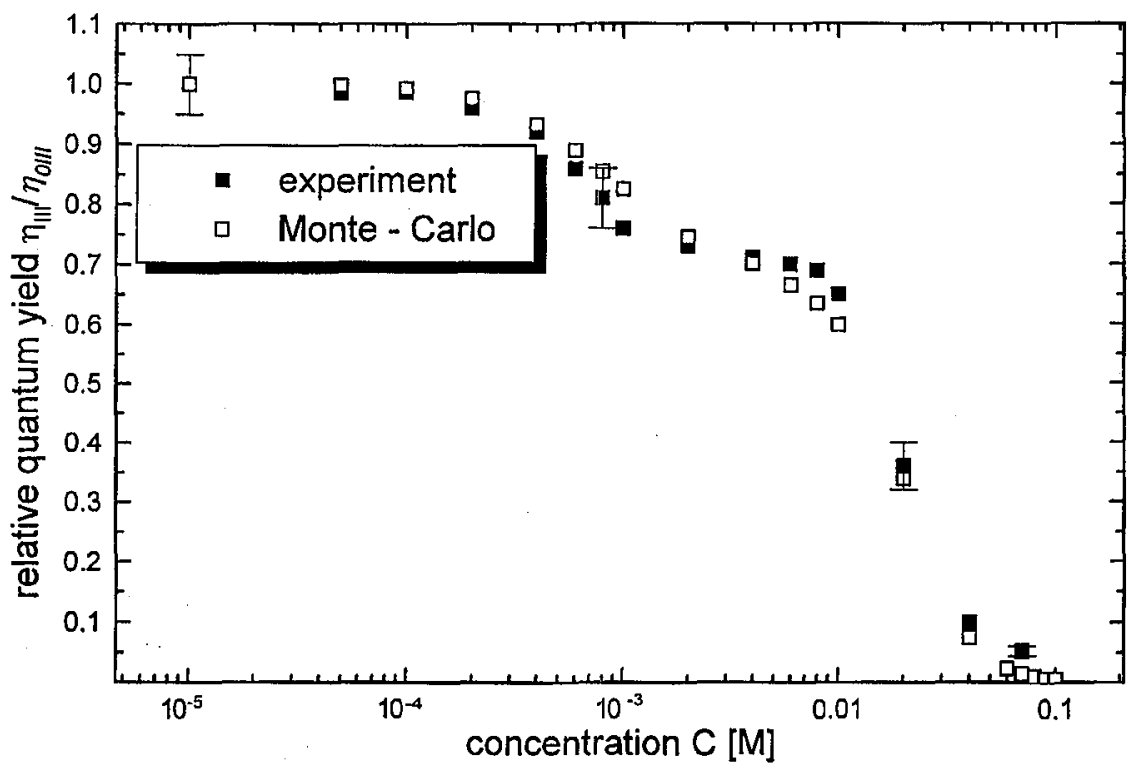

Fig. 2. Concentration dependence of the relative quantum yield for system III (no additives). Full squares denote experimental data whereas the empty ones correspond to the results of Monte-Carlo simulation. 
TABLE II

Critical parameters for energy transport within and between base and acid forms of RB in glycerol-ethanol solution at $293 \mathrm{~K}$ found based on absorption and fluorescence measurements.

\begin{tabular}{l|c|c|c|c}
\hline \hline \multirow{2}{*}{$\begin{array}{l}\text { Critical } \\
\text { parameter }\end{array}$} & \multicolumn{2}{|c|}{ Forward transfer } & \multicolumn{2}{c}{ Reverse transfer } \\
\cline { 2 - 5 } & base-base & base-acid & acid-acid & acid-base \\
\hline$I\left[10^{-13} \mathrm{~cm}^{3} / \mathrm{M}\right]$ & 4.781 & 8.469 & 5.742 & 2.932 \\
$R_{0}[\AA]$ & 56.6 & 62.3 & 55.4 & 49.6 \\
$C_{0}\left[10^{-3} \mathrm{M}\right]$ & 2.18 & 1.64 & 2.33 & 3.25
\end{tabular}

TABLE III

The effect of concentration on the composition of system III. Table contains monomer $\left(C_{\mathrm{M}}\right)$ and dimer $\left(C_{\mathrm{D}}\right)$ concentrations of base and acid forms.

\begin{tabular}{c|c|c|c|c}
\hline \hline$C[\mathrm{M}]$ & $\begin{array}{c}C_{\mathrm{M}}[\mathrm{M}] \\
\text { (base) }\end{array}$ & $\begin{array}{c}C_{\mathrm{D}}[\mathrm{M}] \\
\text { (base) }\end{array}$ & $\begin{array}{c}C_{\mathrm{M}}[\mathrm{M}] \\
\text { (acid) }\end{array}$ & $\begin{array}{c}C_{\mathrm{D}}[\mathrm{M}] \\
\text { (acid) }\end{array}$ \\
\hline $10^{-5}$ & $9.868 \times 10^{-6}$ & $2.62919 \times 10^{-10}$ & $1.31474 \times 10^{-7}$ & $1.72917 \times 10^{-14}$ \\
$5 \times 10^{-5}$ & $4.70039 \times 10^{-5}$ & $5.96529 \times 10^{-9}$ & $2.9841 \times 10^{-6}$ & $8.9051 \times 10^{-12}$ \\
$10^{-4}$ & $8.92042 \times 10^{-5}$ & $2.1485 \times 10^{-8}$ & $1.07526 \times 10^{-5}$ & $1.15618 \times 10^{-10}$ \\
$2 \times 10^{-4}$ & $1.63641 \times 10^{-4}$ & $7.23014 \times 10^{-8}$ & $3.6212 \times 10^{-5}$ & $1.31131 \times 10^{-9}$ \\
$4 \times 10^{-4}$ & $2.87568 \times 10^{-4}$ & $2.23277 \times 10^{-7}$ & $1.11961 \times 10^{-4}$ & $1.25352 \times 10^{-8}$ \\
$6 \times 10^{-4}$ & $3.91442 \times 10^{-4}$ & $4.13711 \times 10^{-7}$ & $2.07645 \times 10^{-4}$ & $4.31164 \times 10^{-8}$ \\
$8 \times 10^{-4}$ & $4.82636 \times 10^{-4}$ & $6.2893 \times 10^{-7}$ & $3.15907 \times 10^{-4}$ & $9.97972 \times 10^{-8}$ \\
$10^{-3}$ & $5.64878 \times 10^{-4}$ & $8.61535 \times 10^{-7}$ & $4.33024 \times 10^{-4}$ & $1.1851 \times 10^{-7}$ \\
$2 \times 10^{-3}$ & $8.97543 \times 10^{-4}$ & $2.17508 \times 10^{-6}$ & 0.001096 & $1.20057 \times 10^{-6}$ \\
$4 \times 10^{-3}$ & 0.00138 & $5.14237 \times 10^{-6}$ & 0.002596 & $6.7401 \times 10^{-6}$ \\
$6 \times 10^{-3}$ & 0.001753 & $8.30184 \times 10^{-6}$ & 0.004195 & $1.75956 \times 10^{-5}$ \\
$8 \times 10^{-3}$ & 0.002069 & $1.15564 \times 10^{-5}$ & 0.00584 & $3.41036 \times 10^{-5}$ \\
$10^{-2}$ & 0.002347 & $1.48681 \times 10^{-5}$ & 0.007511 & $5.64122 \times 10^{-5}$ \\
$2 \times 10^{-2}$ & 0.003433 & $3.18167 \times 10^{-5}$ & 0.015992 & $2.55747 \times 10^{-4}$ \\
$4 \times 10^{-2}$ & 0.004953 & $6.62383 \times 10^{-5}$ & 0.032767 & 0.001074 \\
$7 \times 10^{-2}$ & 0.006604 & $1.1777 \times 10^{-4}$ & 0.056725 & .0 .003218
\end{tabular}

the intermediate concentrations region rapidly from 1 to 0.65 , which corresponds to the values of absolute quantum yields 0.75 and 0.49 , respectively, which are close to those measured for systems I and II over these concentrations. It is seen in the figure that the results of Monte-Carlo simulation describe generally well the character of the quantum yield concentration course (empty squares). The simulated quantum yield exhibits a similar drop at intermediate concentrations, which confirms the assumed mechanism of this anomaly. At concentrations $C \geq 0.01 \mathrm{M}$, where dimers are formed, the values of simulated quantum yield are also close to those of the experiment and the observed discrepancies at certain concentrations 
are mostly within the experimental accuracy limit (error bars). Table IV gives the values of critical parameters like overlap integral $I$, critical distance $R_{0}$ and critical concentration $C_{0}$ for transfer from any kind of monomer to any kind of a dimer. These parameters were used in Monte-Carlo simulation. Mention should be made that in the simulation no best fit parameters were used, but it fully relies on the parameters derived in the experiments and listed in Tables I-IV.

TABLE IV

Critical parameters for energy transport between monomers and dimers for RB in glycerol-ethanol solutions at $293 \mathrm{~K}$. These parameters were found based on derived absorption spectra of dimers and monomer fluorescence spectra.

\begin{tabular}{l|c|c|c|c}
\hline \hline $\begin{array}{l}\text { Critical } \\
\text { parameter }\end{array}$ & $\begin{array}{c}\text { Acid monomer - } \\
\text { acid dimer }\end{array}$ & $\begin{array}{c}\text { Acid mon. - } \\
\text { base dimer }\end{array}$ & $\begin{array}{c}\text { Base mon. - } \\
\text { base dimer }\end{array}$ & $\begin{array}{c}\text { Base mon. - } \\
\text { acid dimer }\end{array}$ \\
\hline$I\left[10^{-13} \mathrm{~cm}^{3} / \mathrm{M}\right]$ & 3.166 & 3.782 & 4.788 & 4.251 \\
$R_{0}[\AA]$ & 50.2 & 51.7 & 56.7 & 55.5 \\
$C_{0}\left[10^{-3} \mathrm{M}\right]$ & 3.13 & 2.87 & 2.18 & 2.31
\end{tabular}

Independently, concentration courses of the quantum yield were also measured for separate forms (results not shown). As expected, no anomalies were found at intermediate concentrations and the quantum yield stayed practically constant up to $C \approx 4 \times 10^{-3} \mathrm{M}$ for system I and up to $C \approx 8 \times 10^{-3} \mathrm{M}$ for system II. The quantum yield remains constant over intermediate concentrations in systems I and II which means that energy migrates homogeneously within the set of single ionic form molecules. At higher concentrations a rapid drop in the quantum yield in both systems due to the quenching by dimers was found. These regularities also confirm earlier remarks.

\section{Final remarks and conclusions}

The results of concentration quenching show that the effect of excitation energy transport in the system investigated is important and that the course of the quantum yield can be satisfactorily described by taking this effect into account. The observed rapid drop of quantum yield at moderate and high concentrations cannot be explained only by the change in $\mathrm{pH}$. However, the mechanism of energy transport is in this case so complicated that it is not possible to compare directly the obtained experimental data with any existing theory. Such a theory should take into account both forward and reverse excitation energy transport, direct excitation of both forms and the trapping by dimers. However, it was possible to reproduce the experimental course of quantum yield by means of Monte-Carlo method.

Since in the system investigated the base-acid equilibrium constant is much higher than that for dimerization it is possible to distinguish between both effects in the concentration scale. Of course, such a relation may not be always fulfilled, since it strongly depends on solvent, temperature, pressure or the dye investigated. 
In further studies we plan to deal with the explanation of a similar anomaly observed for mean fluorescence lifetimes [22] and to adopt a self-consistent diagrammatic method to the problem herein investigated.

\section{Acknowledgment}

This work was supported by the grant BW 5200-5-0191-8. Dr. P. Bojarski would like to thank the DAAD for the photomultiplier granted. We would like to thank TASK center for the use of their computers. We are grateful to Mrs. A. Jankowicz for technical help.

\section{References}

[1] C. Bojarski, Z. Naturforsch. A 39, 948 (1984).

[2] R. Twardowski, J. Kuśba, Z. Naturforsch. A 43, 627 (1988).

[3] K. Sienicki, W.L. Winnik, Chem. Phys. 121, 163 (1988).

[4] K. Sienicki, W.L. Mattice, J. Chem. Phys. 90, 6178 (1989).

[5] L. Kułak, C. Bojarski, J. Fluoresc. 2, 123 (1992).

[6] L. Kułak, C. Bojarski, Chem. Phys. 178, 113 (1993).

[7] L. Kułak, C. Bojarski, Chem. Phys. 191, 43 (1995).

[8] L. Kułak, C. Bojarski, Chem. Phys. 191, 67 (1995).

[9] P. Bojarski, L. Kułak, Chem. Phys. 220, 323 (1997).

[10] C. Bojarski, G. Żurkowska, Z. Naturforsch. A 42, 1451 (1987).

[11] P. Bojarski, A. Kawski, J. Fluoresc. 2, 99 (1992).

[12] P. Bojarski, A. Kawski, B. Kukliński, H. Diehl, Z. Naturforsch. A 49, 931 (1994).

[13] P. Bojarski, J. Lumin. 71, 47 (1997).

[14] P. Bojarski, A. Matczuk, C. Bojarski, A. Kawski, B. Kukliński, G. Żurkowska, H. Diehl, Chem. Phys. 210, 485 (1996).

[15] P. Bojarski, Chem. Phys. Lett. 278, 225 (1997).

[16] P. Bojarski, L. Kułak, Asian J. Spectrosc. 1, 107 (1997).

[17] J. Ferguson, A.W.H. Mau, Chem. Phys. Lett. 17, 543 (1972).

[18] J. Ferguson, A.W.H. Mau, Aust. J. Chem. 26, 1617 (1973).

[19] L.V. Levshin, T.D. Slavnova, V.I. Jushakov, N.B. Zorov, V.Z. Pastchenko, Zh. Fiz. Chim. 48, 84 (1974).

[20] G. Obermueller, C. Bojarski, Acta Phys. Pol. A 52, 431 (1977).

[21] P.J. Sadkowski, G.R. Fleming, Chem. Phys. Lett. 57, 526 (1978).

[22] C. Bojarski, G. Żurkowska, J. Tyrzyk, Z. Naturforsch. A 37, 74 (1982).

[23] R.S. Moog, M.D. Ediger, S.G. Boxer, M.D. Fayer, J. Phys. Chem. 86, 4694 (1982).

[24] I. Lopez Arbeloa, K.K. Rohatgi-Mukherjee, Chem. Phys. Lett. 128, 474 (1986); ibid. 129, 607 (1986).

[25] F. Lopez Arbeloa, P. Ruiz Ojeda, I. Lopez Arbeloa, Chem. Phys. Lett. 148, 253 (1988).

[26] T. Lopez Arbeloa, M.J. Tapia Estevez, F. Lopez Arbeloa, I. Urretxa Aguirresacona, I. Lopez Arbeloa, J. Lumin. 48\&49, 400 (1991). 
[27] I. Ketskemety, J. Dombi, R. Horvai, J. Hevesi, L. Kozma, Acta Phys. Chem. (Szeged) 7, 17 (1961).

[28] V.L. Levshin, E.G. Baranova, Opt. Spectrosc. 6, 31 (1959).

[29] Th. Förster, E. König, Z. Elektrochem. Ber. Bunsenges., Phys. Chem. 61, 344 (1957).

[30] A.R. Monahan, D.F. Blossey, J. Phys. Chem. 74, 4014 (1970).

[31] A. Kawski, G. Piszczek, B. Kukliński, T. Nowosielski, Z. Naturforsch. A 49, 824 (1994).

[32] P.P. Feofilov, The Physical Basis of Polarized Emission, Consultant Bureau, New York 1961.

[33] R.E. Dale, R.K. Bauer, Acta Phys. Pol. A 40, 853 (1971).

[34] A. Kubicki, Tech. Experim. Phys. 37, 329 (1989). 